\title{
No Communication Nodes Synchronization for a Low Power Consumption MAC Protocol in WSN based on IR-UWB
}

\author{
Anouar Darif, Rachid Saadane, and Driss Aboutajdine
}

\begin{abstract}
Synchronization is an important issue in multi hops Wireless Sensor Networks (WSN). Such networks are known by their limited resources of energy, storage, computation, and bandwidth. In addition if the networks entities are deployed with high density, it makes the synchronization mandatory for these networks. Impulse Radio Ultra Wide Band (IR-UWB) technology is a promising solution for this kind of networks due to its various advantages such as its robustness to severe multipath fading even in indoor environments, its low cost, low complexity, and low power consumption. To exploit the specific features of this technology, we need a convenient MAC protocol. WideMac was presented as a new low power consumption MAC protocol designed for WSN using IR-UWB transceivers. Because of the luck of synchronization in this protocol, this paper presents a solution for the synchronization problem especially in the case of no communication between the Network's nodes. To implement and evaluate the proposed synchronization mechanism, we used MiXiM platform under OMNet++ Simulator.
\end{abstract}

Keywords- WSN, IR-UWB, WideMac, Synchronization, Packets delivery Ratio, Power Consumption, MiXiM.

\section{INTRODUCTION}

Wireless Sensor Networks (WSNs) may be defined as a set of smart devices, called sensors, which are able to sense and transmit information about their environment on which they are deployed. These devices collect information for users interested in monitoring and controlling a given phenomenon and transfer them to a central point called sink node. This transfer can be done via a set of nodes. In this case we have a multi hop network. The sink node makes the information available to a gateway where the users can access via Internet. So as to obtain information, users use applications that communicate with the network through queries. WSN have been used for many applications, including military sensing [1], agriculture [2], environmental monitoring [3], multimedia [4], Intelligent Vehicular Systems [5]. An illustration of a WSN is presented in Fig.1.

In multi hop networks, nodes synchronization is a big deal due to the crucial role that plays every node in the network to transfer the incoming messages to their destination. If communication in a portion of the network is broken, the whole region depending on this portion is unavailable.

Manuscript received March 29, 2014; revised May 30, 2014.

Authors are with Federal LRIT-GSCM Associated Unit to CNRST (URAC 29), FSR Mohammed V University, Rabat, Morocco, and SIR2C2S/LASIEHTP, Hassania School of Public Labors, Casablanca, Morocco. E-mails: anouar.darif@gmail.com, rachid.saadane@ehtp.ac.ma, aboutaj@fsr.ac.ma.

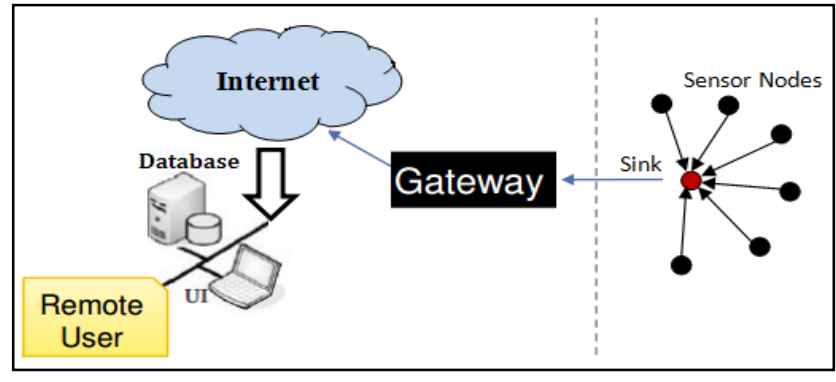

Fig. 1. Sensor network architecture.

New wireless technologies emerge in the recent few years, providing large opportunities in terms of low power consumption, high and low rate and are promising for environment monitoring applications. IR-UWB technology is one of these new technologies and is considered as a next generation of the IEEE802.15.4 standard. It has proven an important technique for supporting several features such as low-power communication, short-range, robustness against interference and mobility. Its introduction in the field of Wireless Sensor Network (WSN) is promising for researchers especially for its low power consumption feature [6]. To take full advantage of this new technology (IR-UWB), it is necessary to develop new protocols for the MAC layer that are relevant and that can take into account the inability to implement the clear channel Assessment (CCA) mechanism as well as other challenges.

WideMac was presented as a novel Medium Access Control (MAC) protocol designed for wireless sensor networks using impulse radio ultra wide band transceivers. Its low power consumption is the main advantage that leads it to be very close to an ideal energy consumption model for the IR-UWB based transceivers. It manages nodes active an inactive periods by keeping them in their sleeping mode the majority of time during each time window (Tw). As a result, nodes are active only during the activity time (Ta) which has to be smaller compared to Tw. In order to transmit a packet, a source node in its activity period must wait to receive the beacon of the destination node. If the source node fails to receive this beacon (especially when the destination node's beacon is transmitted outside the active period of the source node), a breakdown in communication between the two nodes is caused in one direction or in both directions. The first problem was overcome in a previous work [7]. The second problem referring to breakdown in communication in both directions is the present work concern. 
The present paper is organized as follows. In Section III we introduce IR-UWB. Section IV presents WideMac Protocol. The Synchronization mechanism implementation is presented in section V. Simulations and results are presented in section VI; finally, Section VII concludes the paper.

\section{RELATED WORK}

Since its introduction in the field of WSN, IR-UWB is a very promising technology. Several IR-UWB MAC-PHY models have been proposed. Un-Slotted and Slotted ALOHA were the first proposed MAC protocols to go with the new IEEE802.15.4a standard where in [8] an analytical modeling is presented, and in [9] an additional performance evaluation of slotted ALOHA over IR-UWB using an existing simulation platform is also presented. New ALOHA-like protocols and their benefits are also presented in [10]. Multichannel distributed protocols: M-ALOHA, MPSMA, BSMA and their performances evaluation are presented in [11].

All these works did not focus on power optimization and efficiency issue. The authors of [12] introduced a low power and routing friendly MAC protocol for Impulse Radio Ultra Wideband sensor networks. It was the first work dealing with the power issue. In their proposed protocol called WideMac, the synchronization of the nodes' wakeup periods was not considered. This lack of synchronization has a huge impact in the network's performance and throughput. In our previous recent work [7] we studied the one direction communication problem. Unfortunately, it wasn't the only synchronization problem to deal with in this protocol. We observed a second situation where there is no communication between some nodes in both directions. This paper comes to solve this problem and presents the full synchronization mechanism to be added to this protocol. Also, we propose a detailed presentation of this synchronization problem, and a simulation evaluation of the implemented synchronization mechanism.

\section{IMPULSE RADIO ULTRA WIDE BAND}

\section{A. IR-UWB Signal}

The IR-UWB signal uses pulses baseband a very short period of time of the order of a few hundred picoseconds. These signals have a frequency response of nearly zero hertz to several GHz. According to [13] there is no standardization, the waveform is not limited, but its features are limited by the FCC mask. There are different modulation schemes baseband for IR-UWB [14]. This paper uses the PAM technique for IRUWB receiver. As shown in Fig. 2, signals are transmitted in form of very short pulses with every symbol is transmitted over $T_{s}$ period that consists of $N_{f}$ pulses over $N_{f}$ frames (one pulse per frame) [15]. Every frame of duration $T_{f}$ contains $N_{C}$ chips. The symbol waveform of duration $T_{s}=T_{f} N_{f}$ is given by:

$$
Y_{T}(t)=\sum_{j=0}^{N_{f}-1} Y\left(t-j T_{f}-c_{j} T_{c}\right)
$$

Where: $Y(t)$ is ultra-short pulse with duration $T_{p} \ll T_{f}$. $T_{c}=T_{f} / N_{c}$ is the chip duration with a time-hoping (TH) code $\left\{c_{j}\right\} \in\left[0, N_{c}-1\right], j \in\left[0, N_{f}-1\right]$.

We used Pulse Amplitude Modulation (PAM) [16], where the information-bearing symbols $s[n] \in\{ \pm 1\}$ are modeled as binary independent and identically distributed with energy $\varepsilon_{s}$ spread over $N_{f}$ frames and the symbol waveform has unit energy $\left(\int Y_{T}^{2}(t) d t=1\right)$.

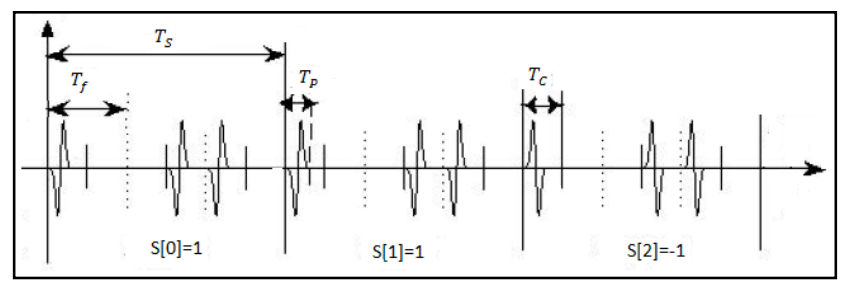

Fig. 2. TH-UWB signal with PAM modulation

The transmitted UWB waveform is then given by:

$$
u(t)=\sqrt{\varepsilon_{s}} \sum_{n=0}^{\infty} s[n] Y_{T}\left(t-n T_{s}\right)
$$

$u(t)$ propagates through a multipath channel, whose impulse response represented in (3) has coefficients $\alpha_{l}$ and delays $\tau_{l}$, obeying $\tau_{l}<\tau_{l+1}$.

$$
h(t)=\sum_{l=0}^{L-1} \alpha_{l} \delta\left(t-\tau_{l}\right)
$$

$\tau_{0}$ is the timing offset refers to the first arrival time. To isolate $\tau_{0}$, we define $\tau_{l, 0}=\tau_{l}-\tau_{0}$ as the relative time delay of each channel tap, where $\tau_{L-0,1}$ is channel delay spread.

To avoid inter-symbol interference (ISI), $T_{f}$ is selected to satisfy the following condition:

$$
T_{f} \geq\left(c_{N_{f}-1}-c_{0}\right) T_{c}+\tau_{L-0,1}+T_{p}
$$

The received pulse within each frame is

$$
Y(t)=\sum_{l=0}^{L-1} \alpha_{l} Y\left(t-\tau_{l, 0}\right)
$$

The waveform in the output of the receiver antenna is:

$$
r(t)=\sqrt{\varepsilon_{s}} \sum_{n=0}^{\infty} s[n] Y_{R}\left(t-n T_{s}-\tau_{0}\right)+w(t)
$$

$Y_{R}(t)$ is the received waveform of each symbol:

$Y_{R}(t)=\sum_{j=0}^{N_{f}-1} Y_{r}\left(t-j T_{f}-c_{j} T_{c}\right)=\sum_{l=0}^{L-1} \alpha_{l} Y\left(t-\tau_{l, 0}\right)$

$w(t)$ represents the pass band filtered zero-mean additive white Gaussian noise (AGWN) with power spectral density (PSD) $N_{0} / 2$ [17] and with double sided bandwidth B $\left(>1 / T_{s}\right)$.

\section{B. IR-UWB Advantages}

There are several features of IR-UWB signals which make them attractive for a wide range of wireless applications. Some of the major advantages of IR-UWB are: low complexity, low power consumption [18], and good time-domain resolution allowing for location and tracking applications. With all these advantages, the IR UWB approach has been selected by the IEEE802.15.4a standard as the alternative PHY for the IEEE802.15.4 standard "in providing communications and high precision ranging/location capability, high aggregate throughput, and ultra low power; as well as adding scalability to higher data rates, longer range, and lower power consumption and cost” [19]. 


\section{WIDEMAC}

\section{A. Presentation}

WideMac is a new MAC protocol designed for WSN based on IR-UWB [12]. It makes all nodes periodically (period $\mathrm{T}_{\mathrm{W}}$, identical for all nodes) and asynchronously wake up, transmit a beacon message announcing their availability and listen for transmission attempts during a brief time $T_{\text {Listen }}$.

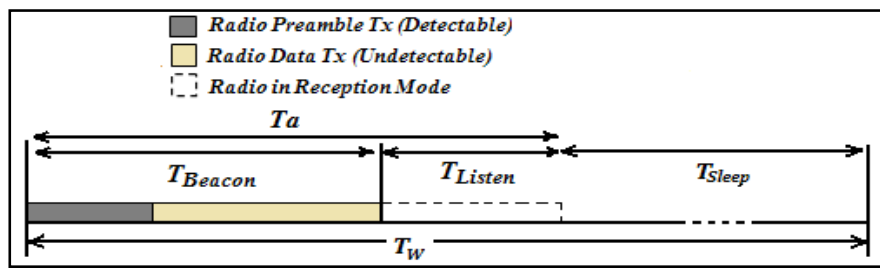

Fig. 3. WideMac period

Fig. 3 illustrates a single period structure. It starts with a known and detectable preamble and is followed by a data sequence which announces the node address and potentially other information, such as a neighbor list or routing table information (for instance, cost of its known path to the sink). A small listening time follows $T_{\text {Listen }}$, during which the node stays in reception mode and that allows it to receive a message. The whole period composed of $T_{\text {beacon }}$ and $T_{\text {Listen }}$ is called $T_{a}$ (time of activity); and it's very small compared to the time window $T_{W}$. This period is followed by a long sleeping period $T_{\text {Sleep }}$ during which nodes save energy by keeping the radio in its sleeping mode.

Nodes' communication process can be described as follow: The sender node waits for the destination node's beacon message during its listening time $\mathrm{T}_{\text {Listen }}$. Once it receives it, it sends data and waits for the acknowledgment message. The later indicates the successful data reception. Fig. 4 describes this process during a single period Tw.

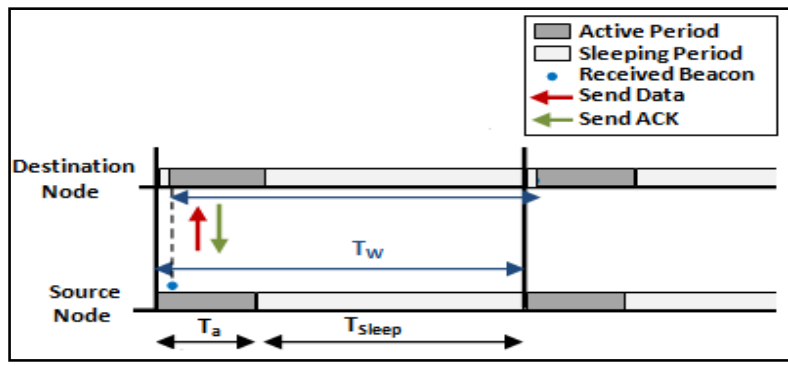

Fig. 4. Normal communication procedure in WideMac Tw

\section{B. Power Consumption}

Each normal $T_{W}$ interval starts with a beacon frame transmission followed by a packet or a beacon reception attempt, during this start a node must enter transmission mode $\left(E_{\text {SetupTx }}\right)$, transmit its beacon $\left(T_{\text {Beacon }} P_{T x}\right)$, switch to reception mode $\left(E_{S W R x T x}\right)$ and attempt a packet reception ( $\left.T_{\text {Listen }} P_{R x}\right)$. These costs are regrouped in the beacon energy $E_{\text {Beacon }}$.

$$
\begin{aligned}
\mathrm{E}_{\text {Beacon }}=\mathrm{E}_{\text {SetupTx }}+ & \mathrm{T}_{\text {Beacon }} \mathrm{P}_{\mathrm{Tx}}+\mathrm{E}_{\text {SwTxRx }} \\
+ & \mathrm{T}_{\text {Listen }} \mathrm{P}_{\mathrm{Rx}}
\end{aligned}
$$

In addition, during a time $L$, a node must sometimes transmit a packet $E_{\text {Trans }}$ or receive one $E_{\text {Recv }}$, and sleep the rest of the time $E_{\text {Sleep }}$, resulting to the following average power consumption:

$$
\mathrm{P}_{\text {WideMac }}=\frac{1}{\mathrm{~T}_{\mathrm{W}}}\left(\mathrm{E}_{\text {Beacon }}+\mathrm{E}_{\text {Trans }}+\mathrm{E}_{\text {Recv }}+\mathrm{E}_{\text {Sleep }}\right)
$$

Where:

$$
\begin{gathered}
\mathrm{E}_{\text {Trans }}=\frac{\mathrm{T}_{\mathrm{W}}}{\mathrm{L}}\left[\mathrm{E}_{\text {SetupRx }}+\mathrm{T}_{\mathrm{BD}} \mathrm{P}_{\mathrm{Rx}}+\mathrm{E}_{\text {SwRxTx }}+\mathrm{T}_{\mathrm{M}} \mathrm{P}_{\mathrm{Tx}}\right. \\
\left.\quad+\mathrm{E}_{\text {SwTxRx }}+\mathrm{T}_{\text {Ack }} \mathrm{P}_{\mathrm{Rx}}\right] \\
\mathrm{E}_{\text {Recv }}=\frac{\mathrm{T}_{\mathrm{W}}}{\mathrm{L}}\left[\left(\mathrm{T}_{\mathrm{M}}-\mathrm{T}_{\text {Listen }}\right) \mathrm{P}_{\mathrm{Rx}}+\mathrm{E}_{\text {SwRxTx }}+\mathrm{T}_{\text {Ack }} \mathrm{P}_{\mathrm{Tx}}\right] \\
\mathrm{E}_{\text {Sleep }}=\left[\mathrm{T}_{\mathrm{W}}-\left(\mathrm{T}_{\text {SetupTx }}+\mathrm{T}_{\text {Beacon }}+\mathrm{T}_{\text {SwTxRx }}+\right.\right. \\
\mathrm{T}_{\text {Listen }}+\frac{\mathrm{T}_{\mathrm{W}}}{\mathrm{L}}\left(\mathrm{T}_{\text {SetupRx }}+\mathrm{T}_{\mathrm{BD}}+2 \mathrm{~T}_{\text {SwRxTx }}+\right. \\
\left.\left.\left.2 \mathrm{~T}_{\mathrm{M}}-\mathrm{T}_{\text {Listen }}+\mathrm{T}_{\text {SwTxRx }}+2 \mathrm{~T}_{\text {Ack }}\right)\right)\right] \mathrm{P}_{\text {Sleep }}
\end{gathered}
$$

Where:

$\mathrm{T}_{\mathrm{M}}$ : is the required time to transmit a message

$\mathrm{T}_{\text {Ack }}$ : is the required time to transmit an acknowledgment message.

$\mathrm{T}_{\mathrm{BD}}$ : is the required time to detect a beacon

$\mathrm{P}_{\mathrm{Rx}}$ : is the radio power in reception mode

$\mathrm{P}_{\mathrm{Tx}}$ : is the radio power in transmission mode

$\mathrm{P}_{\text {Sleep }}$ : is the radio power in sleep mode

\section{Backoff Algorithm}

The backoff algorithm has a major effect on collision, latency and fairness [20]. WideMac periodic beacons allow the sender nodes to get some information on the channel state at the destination. This can be used to reduce the hidden and exposed terminal problems. The WideMac transmission procedure works as follows: a candidate sender node first listens for the receiver node's beacon. Once it finds it, it can either immediately attempt transmission (default for lightly loaded networks) or it can start a backoff timer before sending (this is activated by a flag always Backoff in the beacon). In both cases, the sender node waits for an acknowledgment. If it does not arrive, a retransmission procedure begins. The sender node chooses a random time parameterized by the receiver node's Backoff Exponent (BE) which was broadcast in the beacon, using a binary exponential backoff:

$$
T_{\text {Backoff }}=N_{\text {Backoff }} . T_{W}
$$

$$
\text { where } N_{\text {Backoff }} \in\left[0,2^{B E_{\text {Receiver }}}-1\right] \text {. }
$$

The backoff time is thus a function of the wake-up interval $T_{W}$ and of the channel state at the receiver node, as captured by $B E_{\text {Receiver }}$. Such a receiver-based backoff parameterization was also proposed in IR-UWB. The use of a slotted backoff time based on $T_{W}$ is natural since all candidate sender nodes are synchronized on the receiver node's wake up times: using a fraction of $T_{W}$ would not change anything as the node would not transmit before receiving the destination beacon. Using an integer multiple of $T_{W}$ for the unit backoff duration would increase latency and spread the traffic, but this can also be achieved by adapting the value of $B E_{\text {Receiver }}$ to the traffic conditions [21]. 


\section{No Communication Nodes Problem}

During the transmission phase in each Tw time interval, a source node must wait to receive the beacon of the destination node (see Fig. 4). When the destination node's beacon is transmitted outside the active period of the source node, the later cannot deliver it its data. A breakdown in communication happens between the two nodes from the source node to the destination.

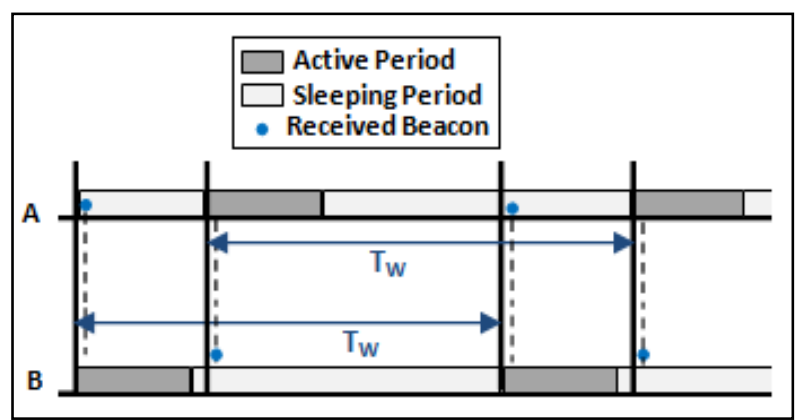

Fig. 5. No communication between node A and B

Worst, Fig. 5 shows the impossible communication case between the two nodes $\mathrm{A}$ and $\mathrm{B}$. In this case, the active periods of the two nodes are disjoint, and no way for them to receive the other's beacon. This is a normal result as the nodes wake up periodically and asynchronously at each static period $T_{W}$ common to all nodes. Therefore a synchronization mechanism between nodes is needed to solve this problem. This synchronization mechanism has to deal with the wakeup time of each node in the network to foster the exchange of data between them and improve the packet delivery ratio as well as throughput. Besides, the neglect of this issue certainly has a cost in terms of performance, efficiency, and throughput of this protocol.

The lack of synchronization between nodes causes a no communication situation between a considerable numbers of nodes among the network as show in Fig.6. It shows the result of a simulation scenario involving 15 nodes as well as the sink node, and are all deployed on a $4 * 4$ grid network. It proves that the nodes 2,11 , and 14 did not send any packets to the sink node in a total number of 100 generated packets. This occurs due to the impossible communication between these three nodes and their neighbors.

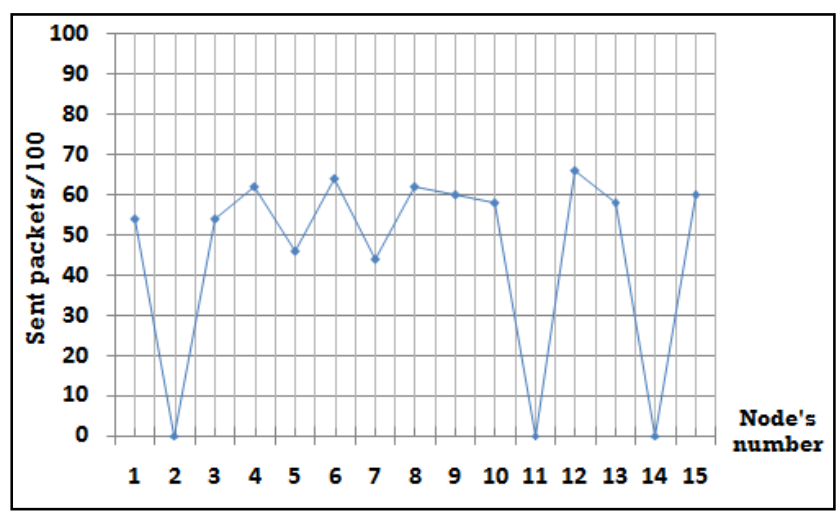

Fig. 6. Packets delivery for the 16 nodes scenario

\section{THE PROPOSED SYNCHRONIZATION MECHANISM}

The synchronization problem solved in our previous work [7] is related to the case when the active periods of the communicating nodes overlap in time. This case can be observed in Fig.4 when we shift the two nodes' role, and assume that the destination node wants to send data to the so called source node in this figure. We can easily conclude that it's impossible as the beacon in this case is received outside the active period of the node which wants to send data. The proposed solution for this problem was based on the exploitation of the beacon that will be received by the destination node. The source node uses it as a synchronization request. Therefore, when the destination node receives the synchronization request, it will decide to perform a synchronization procedure according to the reception time of this request. The operation is done locally in the synchronizeWith() procedure.

In contrast to the previous situation, the problem of no communication discussed in this paper happens when the two active periods of the source and destination nodes are disjoint. Consequently, none of the two nodes will receive the other's request. This situation is presented in Fig. 5. It shows that node A cannot send its data to node B and vice versa, since none of them can receive the beacon of the other. Accordingly, both nodes will remain in this situation of non communication although the first synchronization procedure is used.

In this paper, the proposed solution is based on limiting the number of the synchronization requests to be generated by the source node. It introduces the use of a maximum number MSR (Max Synchronization Retries) to limit the number of these synchronization requests. Therefore, we use a local variable nbrSynchReq as a request counter. The limitation of the generated synchronization requests inside the synchronizeWith() procedure is presented in Algorithm I. In this procedure, if the MSR number is reached, it means that the current node has to launch a synchronization attempt that tries to synchronize the two active periods of the concerned nodes. In other words, the failure of the first synchronization procedure after MSR requests will trigger the second synchronization attempt. Fig. 7 shows that the source node in this case, will schedule the time of its next wake up to Tw- $\beta$ instead of the normal schedule Tw. With this time lag $\beta$, the two nodes' active periods will have more chance to intersect resulting to a possible communication.

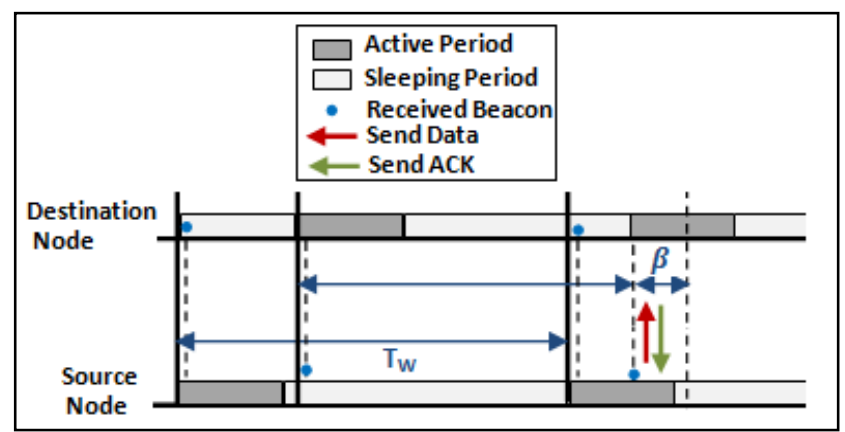

Fig. 7. Synchronization during the situation of no communication

Since the first procedure always fails in the situation of no communication, as a result, the second synchronization 
procedure will be repeated while we are in the no communication situation. This repetition will certainly result in the expected successful communication even if the proposed synchronization mechanism fails the first time.

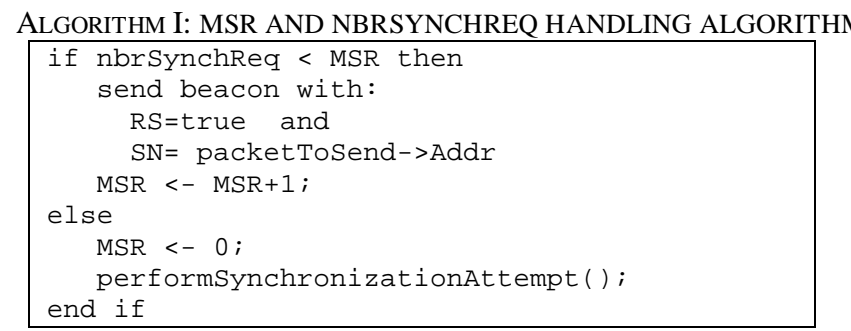

\section{A. Synchronization Parameters}

As described above, $T_{W}$ is the characteristic wake up period of WideMac. $T_{a}$ is the nodes' activity time during which they are waiting for a data message or a beacon message. The parameter maxTxAttempts represent the maximum number of frame retransmission and macAckWaitDuration is the time to wait for an acknowledgement after transmitting a unicast frame. MSR (Maximum Synchronization Request) is the maximum number to request synchronization with a node; when MSR is reached, a synchronization retry is performed with the $\beta$ parameter. It is the time lag for the wake up moment to be retrieved to seek communication in no-communication situation. MSS (Maximum Synchronization Sending) is the maximum number of beacons to generate with a $I S$ flag set to true after a synchronization process. maxBroadcastDataTx is the maximum number of data packets for broadcast to transmit during a $T_{W} \cdot \min B E$ and $\max B E$ are respectively the minimum and the maximum backoff exponent used in the backoff algorithm. For WideMac synchronization parameters value see Table I.

TABLE I. WIDEMAC SYNCHRONIZATION PARAMET
\begin{tabular}{|c|c|}
\hline Parameter & Value \\
\hline Tw & $0.037 \mathrm{~s}$ \\
\hline Ta & - \\
\hline$\beta$ & - \\
\hline MSR & 6 \\
\hline MSS & 14 \\
\hline maxTxAttempts & 20 \\
\hline macAckWaitDuration & $0.0003 \mathrm{~s}$ \\
\hline minBE & 1 \\
\hline maxBE & 6 \\
\hline
\end{tabular}

\section{B. Required beacon's structure}

Our beacon message contains the following fields (as shown in Table II):

TABLE II: SimPLiFIED BEACON STRUCTURE

\begin{tabular}{|c|c|c|}
\hline Field & Type & Signification \\
\hline BE & Integer & Backoff Exponent \\
\hline SN & String & Synchronized Node \\
\hline AB & Boolean & Always Backoff \\
\hline IS & Boolean & Is Synchronized \\
\hline RS & Boolean & Requires Synchronization \\
\hline
\end{tabular}

$B E$ : The backoff exponent number is used to regulate traffic and avoid collisions. It is a parameter in the backoff algorithm and it is used when a node want to transmit a packet.
$S N$ : is the node's address with which the transmitter node of the beacon is synchronized. It is used when the IS flag is true. $A B$ : it is a flag used to run backoff timer always when its value is set to true. It is set to true in highly loaded networks.

IS: it is a flag used to indicate that the beacon's source node is synchronized or not with the node SN.

$R S$ : it is a flag used to indicate that the beacon's sending node is requesting synchronization with the node $\mathrm{SN}$.

As an example of the use of these beacon fields, the algorithm which handles the beacon reception event is described below. It uses the following local variables: nbrSynchRetry, synchSrc, and scheduledSlot. The first one count the number of synchronization attempts with a node. It is increased by 1 when sending a beacon with RS flag set to true. And in each successful synchronization operation it is reset to -1 . The second one marks the last node address to which the current node is synchronized. The third local variable scheduledSlot serves to schedule the packet transmission window. Also, in this algorithm we use the flowing procedures: transmitPacket() and synchronizeWith(). The former performs a packet transmission to the node from which we get the beacon. The latter, performs a synchronization operation with the received beacon node. This operation depends on the beacon reception time and the parameter $\beta$.

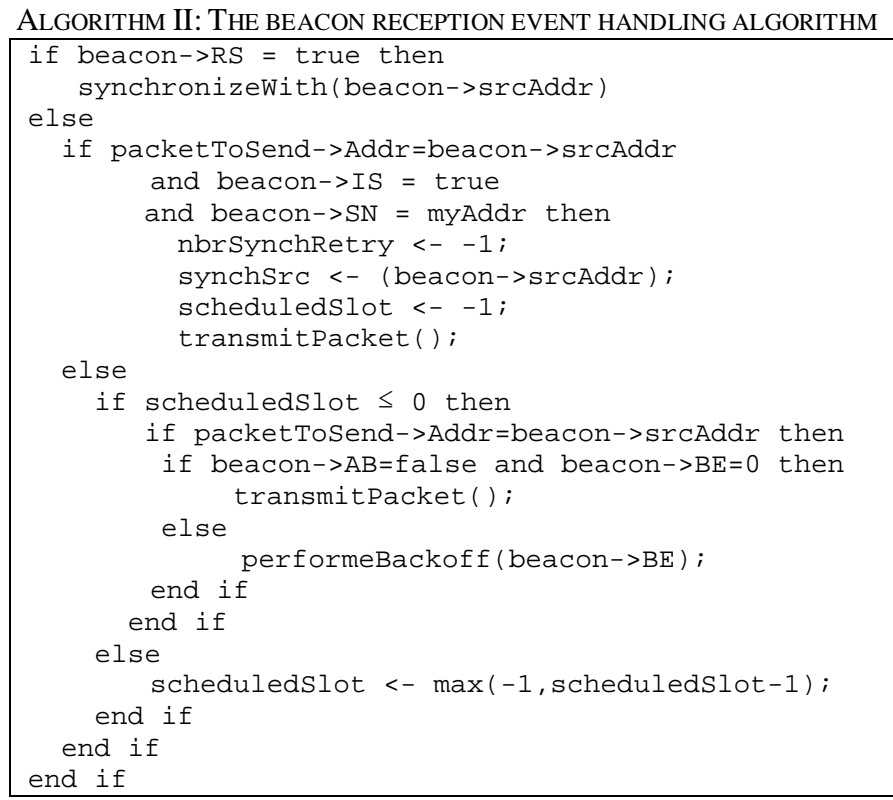

\section{SimULATION AND RESULTS}

\section{A. Simulation Platform}

$\mathrm{OMNeT}++$ is an extensible, modular, component-based $\mathrm{C}++$ simulation library and framework which also includes an integrated development and a graphical runtime environment; it is a discreet events based simulator and it provides a powerful and clear simulation framework [22]. MiXiM joins and extends several existing simulation frameworks developed for wireless and mobile simulations in $\mathrm{OMNeT++}$. It provides detailed models of the wireless channel, wireless connectivity, mobility models, models for obstacles and many communication protocols especially at the Medium Access Control (MAC) level. Moreover, it provides a user-friendly 
graphical representation of wireless and mobile networks in OMNeT++, supporting debugging and defining even complex wireless scenarios [23].

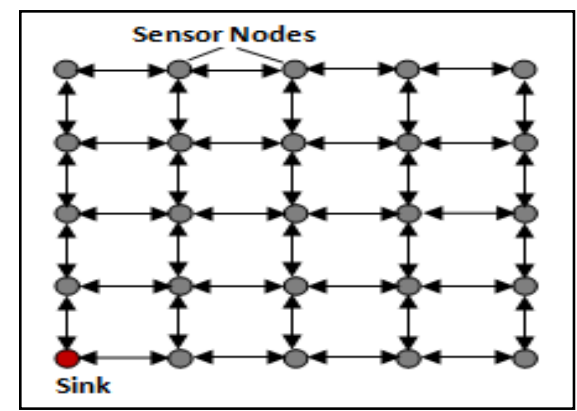

Fig. 8. The 25 nodes' number scenario

We used a grid based topology, where nodes transmit packets to a Sink node. Fig. 8 shows the network structure chosen in the 25 nodes' number scenario, with a sink node in the corner.

\section{B. Energy and Timing Parameters}

We run the simulations under MiXiM2.1 release framework with the OMNeT++ 4.2 network simulator witch the network structure shown in Fig. 8 with different parameters' values to evaluate our proposed Synchronization mechanism. For the energy consumption we used the following radio power consumption parameters (see Table III).

TABLe III. ENERgy PARAMETERS

\begin{tabular}{|c|c|}
\hline Parameter & Value $(\mathrm{mW})$ \\
\hline $\mathrm{P}_{\mathrm{Rx}}$ & 36.4 \\
\hline $\mathrm{P}_{\mathrm{Tx}}$ & 1.212 \\
\hline $\mathrm{P}_{\text {Sleep }}$ & 0.12 \\
\hline $\mathrm{P}_{\text {SetupRx }}$ & 36.4 \\
\hline $\mathrm{P}_{\text {SteupTx }}$ & 1.212 \\
\hline $\mathrm{P}_{\text {SwTRx }}$ & 36.4 \\
\hline $\mathrm{P}_{\text {SwRxTx }}$ & 36.40 \\
\hline
\end{tabular}

For the radio timing we used the parameters shown bellow in Table IV.

Table IV. Timing Parameters

\begin{tabular}{|c|c|}
\hline Parameter & Value \\
\hline $\mathrm{T}_{\text {SetupRx }}$ & $0.103 \mathrm{~ms}$ \\
\hline $\mathrm{T}_{\text {SetupTx }}$ & $0.203 \mathrm{~ms}$ \\
\hline $\mathrm{T}_{\text {SwTxRx }}$ & $0.120 \mathrm{~ms}$ \\
\hline $\mathrm{T}_{\text {SwRxTx }}$ & $0.210 \mathrm{~ms}$ \\
\hline $\mathrm{T}_{\text {RxToSleep }}$ & $0.031 \mathrm{~ms}$ \\
\hline $\mathrm{T}_{\text {TxToSleep }}$ & $0.032 \mathrm{~ms}$ \\
\hline Bit rate & $0.85 \mathrm{Mbps}$ \\
\hline
\end{tabular}

\section{Results}

\section{1 Ta and $\beta$ Parameters}

Ta and $\beta$ are considered as the key parameters for our proposed synchronization mechanism, since there values influence directly the network's performance and throughput. A study of their impact on the packets delivery ratio has been made in order to choose the values that give the best result and increase the efficiency of WideMac protocol. Ta represent the active period during a time window Tw such that $0<\mathrm{Ta} \leq \mathrm{Tw}$. Accordingly, if the value of Ta is near to Tw, the node is more available but with a high power consumption. On the other hand, if $\mathrm{Ta}<<\mathrm{Tw}$, the node is less available but with an optimized power consumption. As a result, the Ta parameter directly influences the power consumption as well as the availability of nodes and has to be chosen with an appropriate value. We chose to study Ta with the following values: $5 \%$, $10 \%$ and $15 \%$ of $\mathrm{Tw}$. The studied max value $T_{a}=15 \% T_{W}$ was adopted to keep acceptable power consumption, since our aim in this work is energy optimization. $\beta$ Values were studied between $1 T_{a}$ to $5 T_{a}$.

We performed simulations with Ta values: $T_{a}=5 \% T_{W}$, $T_{a}=10 \% T_{W}$ and $T_{a}=15 \% T_{W}$ and $\beta$ varying from 1 to 5Ta. Accordingly, $\beta$ values vary from $5 \% \mathrm{~T}_{\mathrm{W}}$ to $75 \% \mathrm{~T}_{\mathrm{W}}$. Results are shown respectively in Fig. 9, Fig. 10 and Fig. 11. The curves of the three figures show the variation of packet delivery ratio according to the $\beta$ parameter. In these figures, we observe that this ratio varies between 39 and 80 percent in Fig. 9. It varies between 49 and 92 percent in Fig. 10. It varies between 42 percent and 100 in Fig. 11. Also, the three figures show that this ratio decreases when the nodes number increase. The decreasing packet delivery ratio with the number of nodes is normal, since the network becomes increasingly loaded and the nodes must contend to synchronize and send data. Also, in all curves we observe that the $\beta$ value which gives the best packets delivery ratio for all Ta values is $\beta=$ $3 T a$. And the $T_{a}$ value that gives more throughputs is $T_{a}=$ $15 \% T_{W}$.

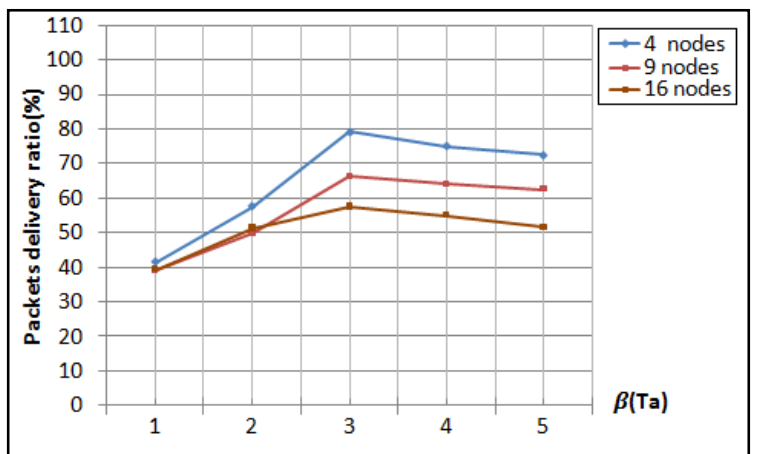

Fig. 9. Packets delivery ratio for $\boldsymbol{T}_{\boldsymbol{a}}=\mathbf{0 . 0 5} * \boldsymbol{T}_{\boldsymbol{w}}$

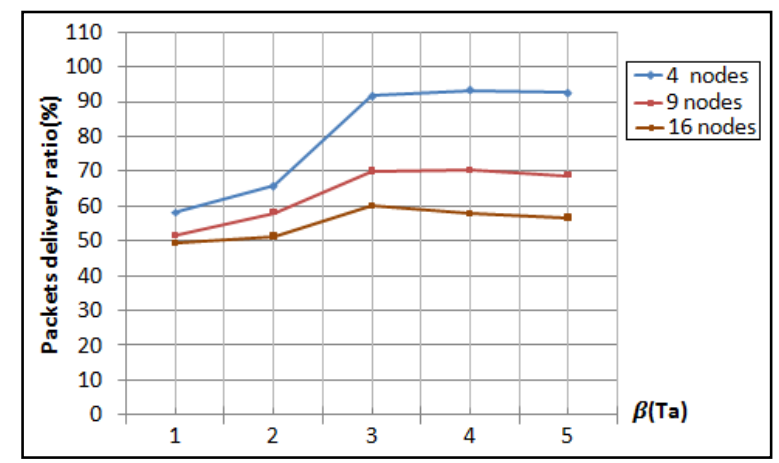

Fig. 10. Packets delivery ratio for $\boldsymbol{T}_{\boldsymbol{a}}=\mathbf{0 . 1} * \boldsymbol{T}_{\boldsymbol{w}}$

\section{C.2 Packets delivery ratio}

To ensure an acceptable quality of service in any MAC protocol packets delivery ratio is a mandatory metric. Accordingly, this section discusses the obtained result concerning this metric. The first part in this section discusses the 16 nodes scenario and the second discusses the packets delivery ratio on several nodes number scenarios. 


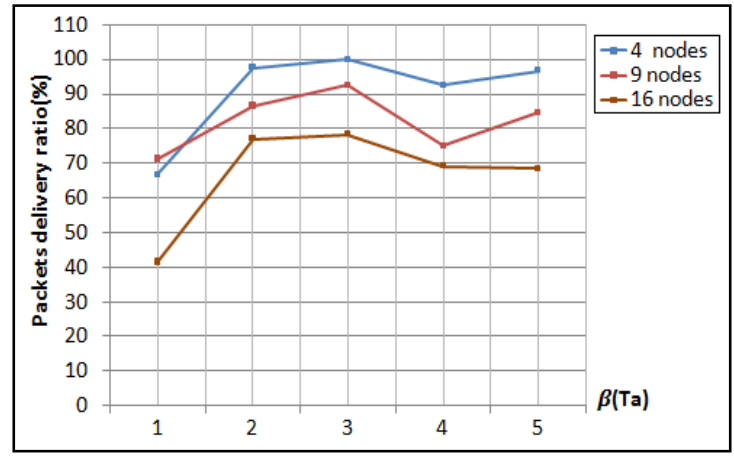

Fig. 11. Packets delivery ratio for $\boldsymbol{T}_{\boldsymbol{a}}=\mathbf{0 . 1 5} * \boldsymbol{T}_{\boldsymbol{w}}$

Section (IV.D) presented the no communication problem between nodes. After the implementation of our proposed synchronization mechanism, Fig. 12 clearly shows that this problem has been successfully resolved since in the same simulation scenario (16 nodes) all nodes have sent more than 55 packets out of 100 . It shows that this synchronization mechanism has solved the no communication problem between the network's nodes. Results are obtained with the synchronized version of WideMac with the following parameters values: $\beta=3 \mathrm{Ta}$ and two values for Ta: $15 \% \mathrm{~T}_{\mathrm{W}}$ and $10 \% \mathrm{~T}_{\mathrm{W}}$. It show also that the value $\mathrm{Ta}=15 \% \mathrm{Tw}$ gives more sent packets compared to the value $\mathrm{Ta}=10 \% \mathrm{Tw}$.

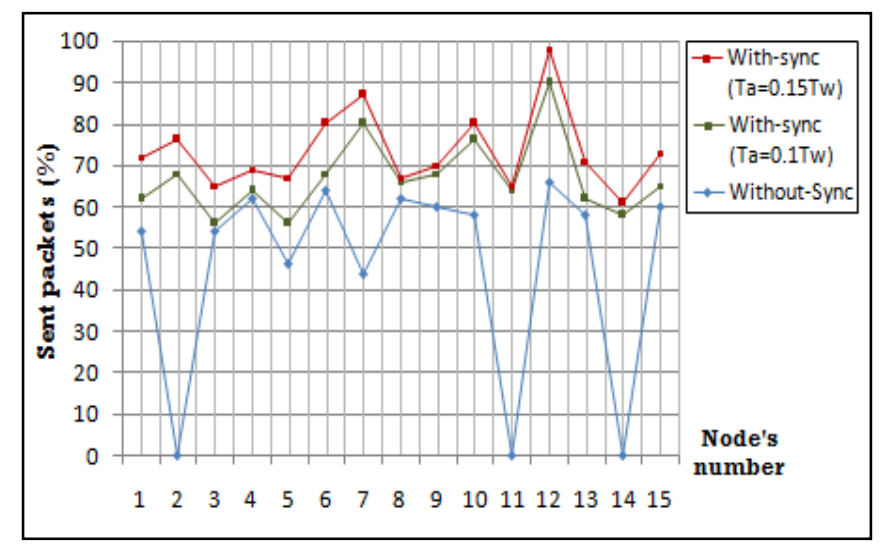

Fig. 12. Packets delivery ratio for the 16 nodes scenario

Fig. 13 shows the simulation results with the synchronized versions of WideMac with the parameters: $\beta=3 T a$ and $\mathrm{Ta}=15 \% \mathrm{~T}_{\mathrm{W}}$. It shows a remarkable improvement in terms of packets delivery ratio in the WideMac protocol after the implementation of both synchronization mechanisms. It shows especially the added value of the proposed synchronization mechanism in this work, as the packets delivery ratio for the full synchronization case exceeds $70 \%$ for 36 nodes scenario, and $93 \%$ for the 9 nodes scenario.

At the same time, the same figure shows a decreasing packets delivery ratio for all cases with the increasing number of nodes. This fact was discussed in the previous section. The remarkable decrease in the performance of the original WideMac protocol with the increasing number of nodes is due to the lack of communication problem as well as the congestion problem.

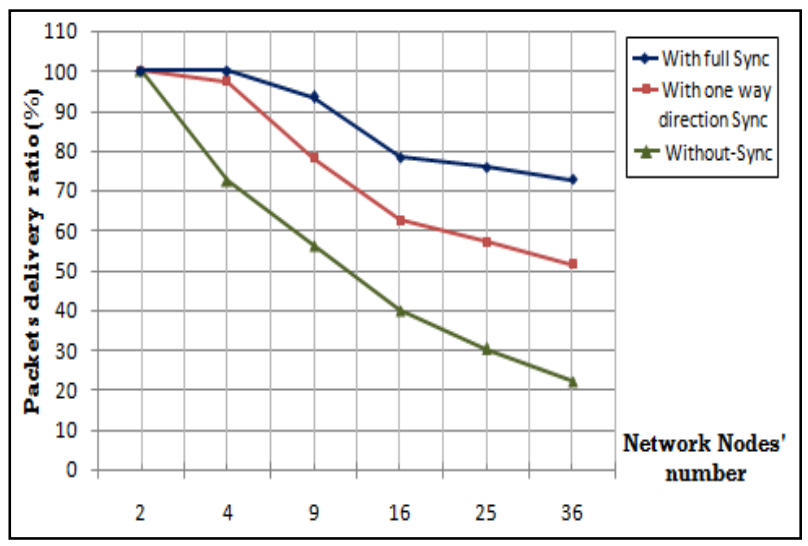

Fig. 13. Packet delivery ratio

\section{C.3 Power Consumption}

Power consumption was and is an interesting issue that is stills a factor in the development of Wireless Sensor Network protocols, especially in the physical and MAC layers. It is the primary metric to design a sensor node in Wireless Sensor Network. It affects directly the lifetime of the network.

In this section, we present the obtained results using the synchronization, timing and energy parameters cited in Sections (V.A) and (VI.B). The optimized power consumption of WideMac protocol with and without synchronization was concretized by the depicted results in Fig. 14. It shows that the power consumption of WideMac with the synchronization mechanism for $T_{a}=10 \%$ and $15 \% T_{w}$ is remarkably less than the original WideMac power consumption. The same figure shows that the consumption in the case $\mathrm{Ta}=20 \% \mathrm{Tw}$ exceeds the original WideMac (without synchronization) consumption. Also, the curves clearly show that the power consumption increases while the Ta value increases. This fact is previously discussed in section (VI.C.1), and we have seen that the active period Ta directly influence nodes' consumption as the nodes' radio is not in its sleep mode. In addition, WideMac was presented as a low power consumption protocol as it keeps nodes in their sleep mode the majority of their time window Tw. Therefore, to have optimal power consumption, we limited nodes' activity period Ta to $15 \% \mathrm{Tw}$.

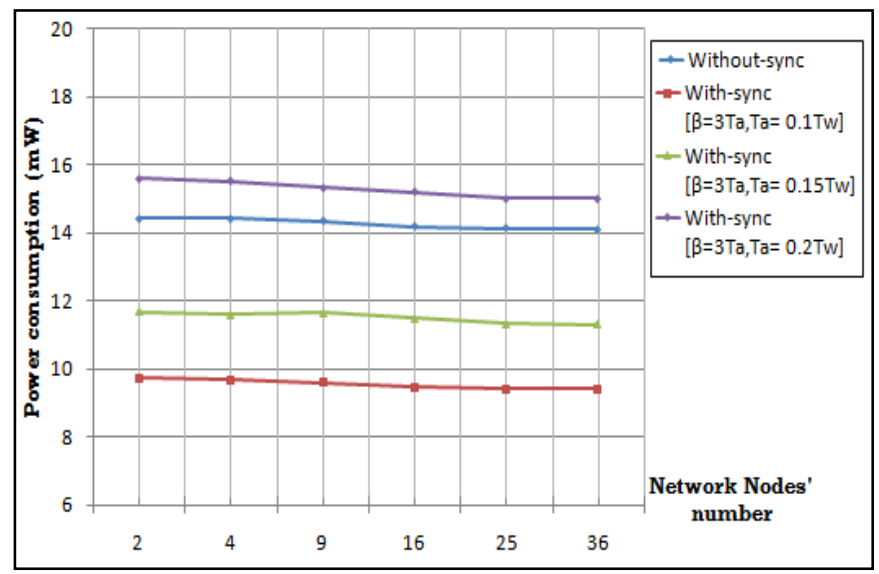

Fig. 14. Power consumption of WideMac with and without synchronization 


\section{CONCLUSION AND FUTURE WORKS}

Medium Access Control layer protocols for Wireless Sensor Network based Impulse Radio Ultra Wide Band must be power efficient to exploit the main features of IR-UWB technology. The low power consumption of WideMac was achieved thanks to the fact that the network nodes are asleep in the $T_{\text {sleep }}$ periods which occupy a wide range in the $T_{W}$ periods; this requires a precise timing between sensor nodes. For these reasons we are addressing the synchronization problem and studying the Packets delivery ratio to ensure an acceptable quality of service in the MAC Layer level. In this work we showed the benefits and the good impact of the synchronization mechanism implemented in WideMac protocol for IR-UWB based WSN. As a future works, we aim to develop a new adapted routing protocol in the network layer that will be paired with the WideMac's synchronization mechanism to largely exploit the WSN based IR-UWB features.

\section{REFERENCES}

[1] L. Sang Hyuk , L. Soobin ; S. Heecheol ; L. Hwang Soo, "Wireless sensor network design for tactical military applications : Remote large-scale environments”, IEEE Military Communications Conference, pp. 1-7, October 2009.

[2] X. Shu-ming, W. Liang-Min, Q. Xiao-qian, Z. Yong-Zhao, "Application Research of WSN in Precise Agriculture Irrigation”, International Conference on Environmental Science and Information Application Technology, pp. 297-300, July 2009.

[3] A. Hua, H. Wangb and J. Wana, "Design of WSN-Based Remote Monitoring System for Environmental Parameters in Substation”, International Journal of Smart Grid and Clean Energy, Vol. 2, No. 1, pp. 113-118, Jan. 2013.

[4] F. Akyildiz, T. Melodia, R. Chowdhury, "A survey on wireless multimedia sensor networks", Journal of Computer networks, Vol. 51, No.4, pp. 921-960, March 2007.

[5] M. Mohamed Nabeel, M. FakhrEl-Dein, S. El-Kader, "Intelligent Vehicle Recognition based on Wireless Sensor Network”, International Journal of Computer Science Issues, Vol. 10, No. 2, pp. 164-175, July 2013.

[6] T. Wang, W. Heinzelman, and A. Seyedi, "Link energy minimization in ir-uwb based wireless sensor networks," IEEE Transactions on Wireless Communication, vol. 9, no. 9, pp. 2800-2811, Sept. 2010.

[7] A. Darif, R. Saadane, D. Aboutajdine, " One Way Direction Communication Synchronization for WideMac Protocol in IRUWB based WSN", International Journal of Computer Networks and Communications Security, Vol. 1, No. 5, pp. 173179, Oct. 2013.

[8] L. D. Nardis, G. Giancola, M.G. D. Benedetto, "Performance Analysis of Uncoordinated Medium Access Control in Low Data Rate UWB networks”, 2nd International Conference on Broadband Networks, pp. 1129-1135, Oct. 2005.

[9] H.-xian Tan, K. Ranjeet Patro, M.-choon Chan, P.-yong Kong, C.-khong Tham, "Performance of Slotted-Aloha over THUWB”, IEEE International Conference on Ultra-Wideband, pp. 868-873, Sept. 2007.

[10] R. Merz, J. Widmer, J.Y. Leboudec, and B. Radunovic, “A Joint PHY-MAC Architecture for Low-Radiated Power TH-UWB Wireless Ad-hoc Networks", Journal of Wireless Communications and Mobile Computing, John Wiley and Sons Vol. 5, pp. 567- 580, Aug. 2005.
[11] N. J. August, W. Chung, and D. S. Ha, "Distributed MAC Protocol for UWB Ad hoc and Sensor Networks”, IEEE Radio and Wireless Symposium, pp 511-514, Jan. 2006.

[12] J. Rousselot, A. El-Hoiydi, J.-D. Decotignie, "WideMac: a low power and routing friendly MAC protocol for Ultra Wideband sensor networks", IEEE International Conference on UltraWideband, pp. 105- 108, Sept. 2008.

[13] A. Lazaro, D. Girbau, R. Villarino, ”Analysis of Vital Signs Monitoring Using an IR-UWB Radar “, Progress In Electromagnetics Research, pp. 265-284, 2010.

[14] N. Rebhi, A. Kachouri, M. Samet and D. Fournier Prunaret, ”TH Differential Pseudo-Random Pulse: A New UWB System for LR-WPAN Applications”, Journal of Telecommunications, Vol. 12, No. 1, pp. 11-16, Jan. 2012.

[15] M. Jing, Z. Nai-tong, "Transmission characteristics analysis of IR-UWB signal”, International Conference on Wireless and Optical Communications Networks, pp. 1-5, April 2009.

[16] T. Valente and A. V. T. Cartaxo, "Distribution of IR-UWB Signals With PAM Modulation in Long-Reach FTTH Networks", Journal of Optical Communications and Networking, Vol. 2, No. 11, pp. 892-900, Nov. 2010.

[17] Y. Park, D. David Wentzloff, "An All-Digital 12 pJ/Pulse IRUWB Transmitter Synthesized From a Standard Cell Library”, IEEE Journal of Solid-State Circuits, Vol. 46, No. 5, pp. 11471157, May 2011.

[18] A. Darif, R. Saadane, D. Aboutajdine, "Performance Evaluation of IR-UWB Compared to Zigbee in Real time Applications for Wireless Sensor Networks", Journal of Convergence Information Technology, Vol. 8, No. 15, pp. 1-12, Oct. 2013.

[19] J. Rousselot, A. El-Hoiydi, and J.-D. Decotignie, "Performance evaluation of the IEEE 802.15.4A UWB physical layer for Body Area Networks", in 12th IEEE International Symposium on Computers and Communications, pp. 969-974, July 2007.

[20] S. Mehta, K. S. Kwak, "A Power Efficient Back-off Scheme for Wireless Sensor Network”, International Journal of Wireless \& Mobile Networks, Vol. 2, No. 1, pp. 15-22, Feb. 2010.

[21] A. Mohammed, S. Mohan, S. Ungarala, A. Gaffar, "Hybrid Binary Exponential Back-Off Mechanism for Wireless Sensor Networks", International Journal of Engineering and Technology, Vol. 5, No. 3, pp. 2391- 2397, Jun-Jul. 2013.

[22] J. Rousselot, J.-D. Decotignie , "An ultra-wideband impulse radio PHY layer model for network simulation”, Transactions of the Society of Modeling and Simulation International, Vol. 87, No. 1-2, pp. 82-112, Jan. 2010.

[23] A.Köpke, M.Swigulski, K.Wessel, D. Willkomm, P.T.Klein Han eveld,T.E.V.Parker, O.W.Visser,H.S.Lichte, S.Valentin, “Simula ting Wireless and Mobile Networks in OMNeT++ The MiXiM Vision”, In Proc. Intl. Workshop on OMNeT++, March 2008.

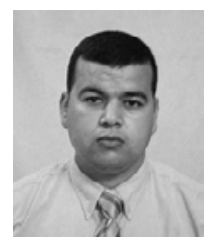

Anouar Darif received the bachelor in EEA (Informatique Electrothecnique, Electronique and Automatique) from Dhar El MahrazFaculty of Sciences at Mohamed Ben Abdellah University Fez, Morocco in 2005. Anouar, received the Diplôme d'Etudes Supérieurs Approfondies in Computer Sciences and Telecommunications from Faculty of Sciences Rabat in 2007. Currently he is doing his $\mathrm{PhD}$ research in Wireless Sensor Network based on ultra wideband tranceivers within the Research Laboratory in Computer Sciences and Telecommunications (GSCM-LRIT) associated with CNRST. His research interests are wireless sensor network, Wireless, Mobile Communication and Computer Sciences. 


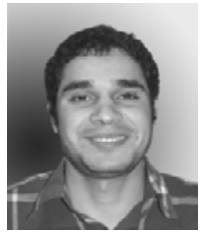

Rachid Saadane received the bachelor in Physic Electronic from Faculty of Science Rabat in 2001. He received the Diplôme d'Etudes Supérieurs Approfondies in Computer Sciences and Telecommunications from Faculty of Sciences of Rabat in 2003. He received the Ph.D. degree in Computer Sciences and Telecommunications from Faculty of Sciences of Rabat in 2007. He is currently a Research and a Teaching Associate in the Electrical Engineering Department at EHTP of Casablanca, Morocco. His research interests include array of UWB channel measurements modeling and characterization, mobile and wireless communications (GSM, WCDMA, TD/CDMA, LTE and LTE-A) digital signal processing for wireless communications systems. Recently, he is intensively interested to the IR-UWB physical layer for WSN and WBAN. Rachid is an active reviewer of various international conferences and journals.

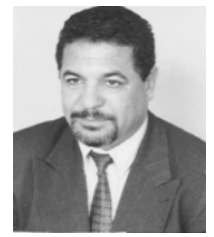

Driss Aboutajdine Received the 3rd Cycle Doctorat and the Doctorat d'Etat in signal processing from the Mohammed V-Agdal University in 1980 and 1985, respectively. He joined Mohammed V-Agdal University on 1978 first as an assistant professor, then as an associate professor in 1985. Since 1990, he is professor with the "Faculté des Sciences" heading the GSCM-LRIT laboratory. Actually he is the national coordinator of a National Information technology network of excellence. Since October 1999 he is IEEE Senior Member and he is Fellow member of TWAS. He has contributed to more than 120 articles to journals and conference proceedings. He was elected member of the Hassan II Moroccan academy of science and technology on May 2006. Curently, he is the head of the Centre National pour la Recherche Scientifique et Technique, Morocco. His research interests include Signal, Image Processing and Computer Sciences. 